\title{
Image Segmentation: Efficacious Processing of Mid - Trimester Fetal Ultrasound Images for Diagnosis of Fetal Aberration Using Global Thresholding Otsu's Method
}

\author{
Yamini $B^{1}$, Premalatha $M^{2}$, Suganya $G^{3}$ \\ ${ }^{1}$ Associate Professor, Department of Computer Science and Engineering, \\ Saveetha School of Engineering, Chennai, India, yamini.subagani@gmail.com \\ ${ }^{2,3}$ School of Computer Science and Engineering, Vellore Institute of Technology, Chennai, India, \\ 12premalatha.m@vit.ac.in, ${ }^{2}$ suganya.g@ vit.ac.in
}

\begin{abstract}
Obstetric ultrasound is a diagnostic medical imaging modus operandi that provides us with the images tool in the care of pregnant women, its detection rate for certain aberrations is still comparatively low due to operator dependency. In our proposed work, Computer aided DSS(decision support system) is projected to detect two structural aberrations namely Spina Bifida and Exomphalos and further classify them. Spina Bifida is a Neural Tube Defect that results in deprived capability to walk, meagre kidney functions and reduced bladder and bowel control. It is of following type: 1)Spina Bifida Occulta, 2) Meningocele defect and 3) Myelomeningocele defect .Exomphalos is the feebleness of baby's abdominal wall due to which the contents of the baby's abdomen obtrudes outside the abdominal wall in the form of a sac. It is of two types- Exomphalos Major and Exomphalos Minor. Our decision support system puts an end to operator dependency and ameliorates diagnostic precision. The proposed DSS has quad phases:1) Pre-processing, 2)Segmentation,3) Feature Extraction and 4) Feature Classification. Through our approach, the detection rate is escalated due to the use of efficient algorithm at every phase of processing the ultrasound images
\end{abstract}

Key words :Spina Bifida Occulta, Meningocele, Myelomenigocele, Exomphalos Major, Exomphalos Minor, Median filter, Otsu's thresholding method.

\section{INTRODUCTION}

Ultrasonography is a medical diagnostic imaging modus operandi that uses elevated occurrence sound energy to envision the clear structure of the body. Three ultrasounds recorded at the three trimesters serve varied purposes. In the first trimester, it is often employed to confirm gestation, to decide the gestational age of the fetus, to assess the due date effectively and to detect ectopic pregnancy. During the second and third trimester, the fetal growth is estimated, multiple fetuses are confirmed, the sex of the unborn baby is determined and continuous check is carried out for the betterment of the fetus and the mother. In addition, it is also used to assess the structural abnormalities or congenital malformations related to the fetus. Obstetric Ultrasonography has eventually become an indispensible obstetric tool in the care of every pregnant woman. Structural aberrations are congenital disorders that exist before birth of the fetus despite of reason. They are categorized by deformities and engross defects in embryonic fetus. The occurrence rates of these aberrations are given in Table1. Detection of these aberrations depends upon the operator performance. We propose a decision support system that takes the anomaly ultrasound (or) second trimester scan images as inputs and automatically detects two structural aberrations such as Spina Bifida and Exomphalos and further classify them. Our decision support system uses Digital image processing techniques to detect the structural aberration in the fetus by processing the fetal ultrasound images. In this manuscript, an computerized decision support system that perceives aberrations of fetus with escalated diagnostic precision by employing efficient image processing algorithms for processing the fetal ultrasound images at every stage.

TABLE 1 : OCCURENCE RATE

\begin{tabular}{|l|l|}
\hline FETAL ABERRATION & OCCURRENCE RATE \\
\hline Spina Bifida & 1.9 per 1000 births \\
\hline Exomphalos & 1 per 4000 births \\
\hline
\end{tabular}

Spina Bifida is a imperfect closing of spine and membranes around the spinal cord caused by Neural Tube Defect (NTD). All the three types of Spina Bifida based on its severity are shown in Figure 1. Among three, Spina Bifida Occulta is less severe and results in small splits in the vertebrae. Since it is the most imperceptible form, the spinal cord does not obtrude. Meningocele is the minimum occurring form of Spina Bifida. Myelomeningocele (or) Open Spina Bifida is the most acute form which results in the exposure of neural contents including the CSF, meninges and nerve roots to obtrude through an aperture. Children with Open Spina Bifida have deprived capability to walk, difficulties with bowel or bladder control and also poor kidney functions. Screening of Open 
Spina Bifida involves 3 different views - coronal plane, traversal plane and sagittal plane. The coronal plane makes sure that an equal amount of foetal tissue is observed in either part of the foetus. Through traversal plane, each vertebra is examined. If any defect is detected in any of the above 2 plane views, then we go for Sagittal plane. All the three types of Spina Bifida are treated soon after the birth of the baby.

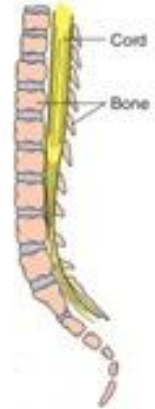

A) NORMAL SPINE

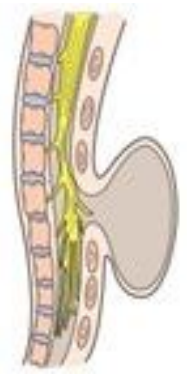

C) MENINGOCELE

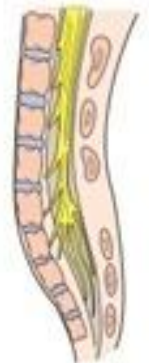

B) SPINA BIFIDA OCCULTA

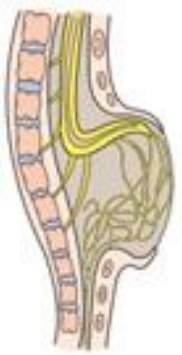

D) MYELOMENINGOCEL

\section{FIGURE 1: TYPES OF SPINA BIFIDA}

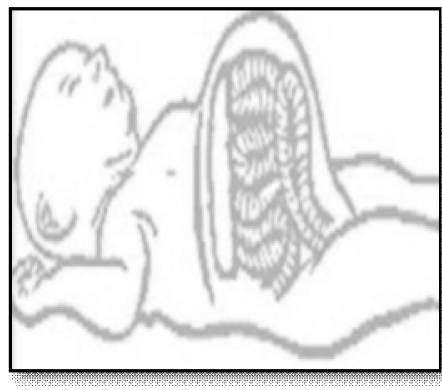

A) EXOMPHALOS MAJOR

\section{FIGURE 2: EXOMPHALOS - TYPES}

Exomphalos (or) Exomphalos is an abdominal wall defect which has a mortality rate of about $25 \%$. It is of two types: Exomphalos Major and Exomphalos Minor which are shown in Figure 2. On account of Exomphalos Major, the digestion tracts, liver and infrequently different organs reside outside the abdomen in a sac which protrudes outside through the navel. On the other hand, in the latter, only the intestine remains in the sac thereby protruding outside through the navel. Due to this abnormality, the abdominal cavity remains small in size. It is common that the intestines project out from stomach into umbilical cord till 10th week of pregnancy, then they return back into fetal stomach. The persistence of the protrusion even during the second trimester confirms Exomphalos. The therapy for defect depends on the size of the sac. If the sac is small (containing only intestines i.e., Exomphalos Minor) and the child is stable, then the surgeon replaces the contents back into the abdominal space and the close the hole at the bottom of the umbilical cord. However, if sac is large (containing intestine along with liver i.e., Exomphalos Major) and the child is unstable, then the doctors place a silo over the intestines which is closed sometime to allow the child to grow so that there is enough space in the abdomen for the obtruded contents.

\section{RELATED WORK}

UmutKonur et al, have developed an automated diagnosis scheme in support of Spina Bifida [1]. In their work, they've taken Trans cerebellar ultrasound images of the fetal head as input and extract fetal skull's curvature scale space (CSS) features. Extracted features are then classified using nearest neighbour $(\mathrm{kNN})$ classification. Primarily, the fetal skull images are fit into elliptical models in-order to confine skull margins and perceive curvature sector of fetal skulls. The CSS features are extracted and CSS images of different contours are compared using CSS Matching algorithm inorder to find a matching score. Further, classification is done based on the matching score and thus the presence of Spina Bifida is detected.

Athira P.K. et al, have developed a DSS for recognition of Congenital Heart Disease by Fetal Ultrasound images [2]. In their work, the ultrasound image of fetal heart is taken as input from which the speckle noise is removed using Patch-based Wiener filter. Then, morphological operations are used for Segmentation. Finally, GLCM (Gray level co-occurrence matrix) is meant for feature mining in order to extract the core features from the image. Based on these features, the images are classified as normal or the presence of congenital heart disease is confirmed using Artificial Neural Network.

UmutKonur et al, developed a computer aided diagnosis for Spina Bifida which takes fetal head ultrasound image as input and pre-processes them by means of thresholding, skeletonization and thinning [3]. The pre-processed images are fitted into ellipse by pseudo - random sample consensus (PRANSAC) thereby retrieving the actual head boundary. The tangent lines slopes at every point of edge were considered for the feature to be extracted where change in between any two consecutive points of an edge depicts abnormality. Based on the extracted feature, simple SVM is used for binary classification. This work provided with only candidate solutions which provoked them to work further and propose another work.

Mahmoud et al, projected a computer aided diagnosis for shaping the type of extended bone fracture shown in x-ray images [4]. Dissimilar versions of noise including Gaussian noise, brightness, Poisson, darkness, and blurring are removed using a range of filters. Then various features are extracted. 
Further, they've used Decision tree, SVM, Neural Networks and Naïve Bayes method for binary classification in order to check if the bone is fractured or not. In addition, they've used Naïve Bayes and Neural Networks for multiclass classification problem in order to find the type of fracture. The performance analysis of every method is measured as Precision, Recall, F-Measure and AUC during the testing phase.

H.D.Cheng et al, suggested a survey for recognition and classification of breast tumour automatically using breast ultrasound descriptions rather than mammogram images [5]. This work was done in-order to eradicate the operator dependency and pick up diagnostic accuracy by automated DSS. Various approaches in every stage of processing the image including pre-processing, image segmentation, feature extraction and selection from image, and image classification were implemented. Finally, results of all different approaches are summarized along with their pros and cons.

\section{PROPOSED METHOD}

We propose a methodical decision support system which detects the fetal aberration by processing the spinal or abdominal ultrasound images of the fetus. The images are processed and are classified if the fetus is normalorabnormal. If the fetus is abnormal and the input was a spinal ultrasound image, then it is classified as whether Spina Bifida Occulta or Meningocele or Myelomeningocele. Of if the input was an abdominal ultrasound, then it is classified as Exomphalos Major or Exomphalos Minor. Finally the fetal aberration is identified exactly. This decision support system assists the Obstetricians in analysing and discerning the aberration in the fetus thereby ameliorating diagnostic precision. It also helps in the eliminating the operator - dependent nature of Ultrasound Imaging.

The various stages involved in the processing of an ultrasound image are given in the Figure3. The fetal ultrasound images of either spine or abdominal region are given as input to the pre-processing stage where the image undergoes two phases De-speckling and Image enhancement. The granular noise, Speckle inherently be real and mortify the feature of ultrasound stills. De-speckling adheres in removing speckle noise without destroying the attributes and quality of the image [6]. In our system, we utilize a median filter for de-speckling as proposed by Mu-Hsein Hsieh et al,[7]. Image Enhancement is the practice of regulating digital images in-order to make them suitable for further [8]. Equalization of histogram is also done for enhancement of image by Nikhil $\mathbf{J}$ et al, [9]. Thus at the finish of pre-processing phase, the feature of the fetal ultrasound image gets improved. The Segmentation phase takes this pre-processed images for further processing.

Superpixels are identified by segmentation process by Qureshi, Q. A., Khan et al,[10]. Depicting images in an easier form for analysing is the target of segmentation analyzed by Alison Noble $\mathbf{J}$ et al, [11].Here we use Otsu's method for segmentation [12]. Otsu's method depicts the minimal collective spread and maximal interclass invariance suggested by Himanshu Makkar et al, [13]. This segmented binary image is a feed to feature extraction module.

In extraction of features, exact interested properties of the image are mined from the fragmented binary image by deciding on ROI (Region of Interest). The features of Binary image are taken into consideration in this phase.

In feature classification phase, initially we use SVM - Binary Classification to classify if the fetus is normal or abnormal by analysing the features extracted in the former phase. Multiclass SVM Classification works on further analysing of abnormal features. SVM procedure deals with notion of optimal hyper plane to discrete two classes of samples. It initially has to be trained using training data samples which are used during classification.

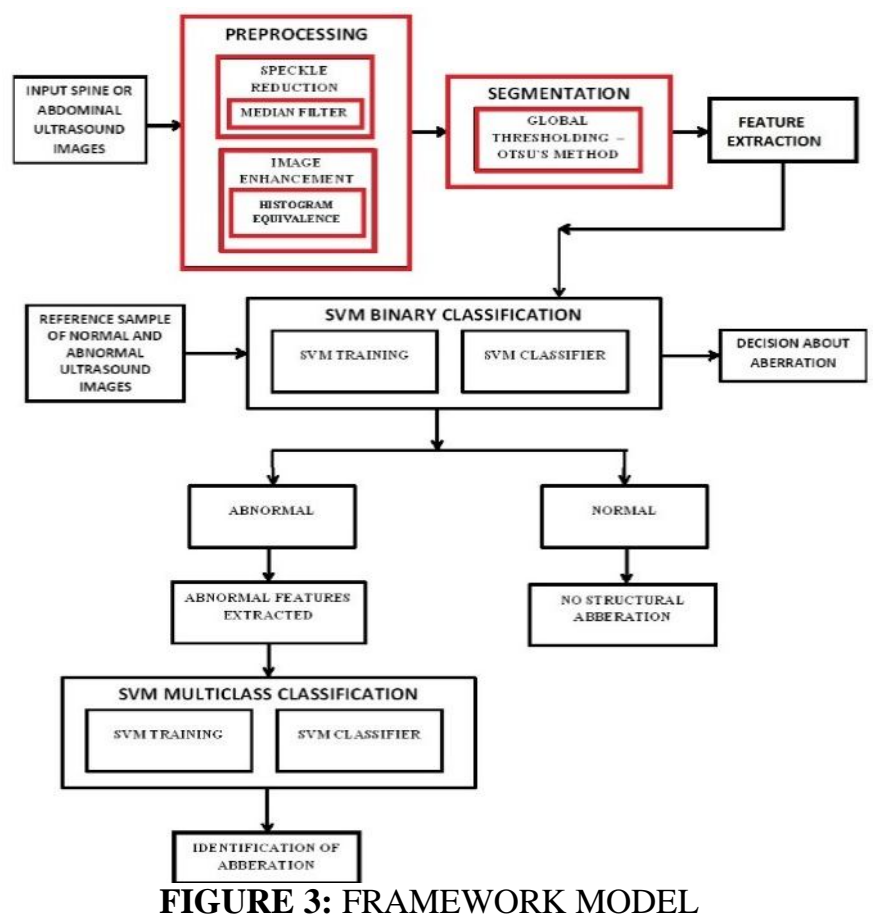

\subsection{PREPROCESSING}

Pre-processing is the primary phase of processing a fetal ultrasound image. The fetal ultrasound samples are composed from Gallery of Fetal-Ultrasound Image. The collected samples include spinal and abdominal images of fetus having Spina Bifida Occulta, Meningocele, Myelomeningocele, Exomphalos Major, Exomphalos Minor and also those that don't have any structural aberrations. The major cons of Ultrasound image are their low contrast along with speckle interference. In order to cope up with these two drawbacks, we go for De-Speckling and image enhancement. De-speckling removes the speckle noise without destroying attributes and quality of image surveyed by Joel T el al, [14]. For De-Speckling we use Median filter as it is proved to be the most efficient filter for de-speckling [15]. It takes the ultrasound image as input, applies noise to removes both the speckle and noise present in image [16]. 
The following algorithm discusses about the steps involved in de-speckling an image using Median filter:

Input : 2D matrix of input noisy image $m * n$.

Method : The input elements of the matrix are padded with 0 's and then sorted as array. The median element of the array is found and is placed in the output matrix.

Output : 2D matrix of Denoised image $m * n$.

Algorithm 1: Median filter

1. Matrix of variant size based on $m$ and $n(m+2 * n+2)$ is assigned with 0 's.

2. Replicate the input matrix into matrix defined in step 1.

3. Generate a $3 \times 3$ window matrix with the values of input matrix.

4. Sort the values of the window matrix by duplicating it in an array.

5. Get the median value in the array values.

6. Consign the median value into output matrix.

7. Do Repeat from step 1 for inclusive input matrix.

The denoised image is then enhanced in the image enhancement phase. Fine-tuning of image for further processing is image enhancement suggested by Mandeep Kaur et al, [17]. We use Histogram equalization for image enhancement suggested by Kuldeep Singh et al, [18].It is a technique used to fine-tune image intensities in order to improve contrast as suggested by Sheng et al, [19]. Histogram equalization generates constant pixel intensity regions. This loom habitually facilitate image improvement idea, since it's simple and comparatively superior to erstwhile outmoded methods [20].

Algorithm 2: defines, steps involved in enhancing the image using histogram equalization:

Input : 2D matrix of greyscale input image $n * m$.

Method: Histogram of key image is compared with equalized histogram to form enhanced output.

Output : 2D matrix of enhanced image $n * m$.

Algorithm 2: Histogram equalization

1. An array $\mathrm{H}$ of length $\mathrm{G}$ is initialized with 0 values where $\mathrm{G}$ represents the gray levels in the key image.

2. As every pixel is scanned, the relevant number of matrix $\mathrm{H}$ is incremented.

3. The cumulative function of the histogram is formed.

4. The cumulative frequency is compared with the equalized histogram.

5. The image is scanned again and the output enhanced image is formed with the new gray levels.

Thus the outcome of the pre-processing phase is an enhanced denoised image which is given as input to the segmentation phase.

Let ' $\mathrm{f}$ ' be a specified image specified by a $\mathrm{r} \mathrm{X}$ c pixel strength matrix vary from 0 to $\mathrm{L}-1$, where ' $\mathrm{L}$ ' is count of likely strength values, habitually 256 and in equation (1) 'p' identifies standardized histogram of ' $f$ ' with a container for each probable intensities. Then,

$$
\mathrm{p}_{\mathrm{n}}=\frac{\text { number of pixels with intensity } \mathrm{n}}{\text { total number of pixels }}
$$

Where $\mathrm{n}=0,1, \ldots \mathrm{L}-1$.

Given $\mathrm{f}$ as input, the histogram equalization $\mathrm{g}$ for an image will be given as in equation (2) and (3),

$$
\begin{aligned}
& \left.\mathrm{g}_{\mathrm{i}, \mathrm{j}}=\operatorname{floor}(((\mathrm{L})-1)) \sum_{\mathrm{n}=0}^{\mathrm{f}_{\mathrm{i} j}} \mathrm{p}_{\mathrm{n}}\right) \\
& \left.\mathrm{W}(\mathrm{k})=\operatorname{floor}((\mathrm{L}))-1) \sum_{\mathrm{n}=0}^{\mathrm{k}} \mathrm{p}_{\mathrm{n}}\right)
\end{aligned}
$$

The inspiration for this conversion comes from opinion of the intensities of ' $\mathrm{f}$ ' and ' $\mathrm{g}$ ' as unbroken arbitrary variables $\mathrm{X}, \mathrm{Y}$ on $[0$ and $L-1]$ with $\mathrm{Y}$ as specified in equation (5) distinct by,

$$
\mathrm{Y}=\mathrm{W}(\mathrm{x})=((\mathrm{L})-1) \int_{0}^{\mathrm{x}} \mathrm{p}_{\mathrm{x}}(\mathrm{x}) \mathrm{dx}
$$

Wherep $_{x}$ is PDF (probability density function) of ' $\mathrm{f}$ '. ' $\mathrm{W}$ ' is collective distributive functionof $X$ repeatedly by $((\mathrm{L})-1)$.

\subsection{IMAGE SEGMENTATION}

In fact, it is a critical phase in the processing of medical images as specified by AlirezaNorouziaet al [21]. The purpose of this phase is to make straightforward in representing image so as to make processing easier suggested by RohanKandwal et al, [22].The pre-processed image is given as input to this phase. There are various methods for performing Image Segmentation such as Region based approach suggested by Mohammed. M et al, [23], Edge Detection based Segmentation [24], Thresholding based Segmentation[25], Feature based Clustering [26] and Artificial Neural Network based Segmentation[27].

In Region based advancement, every pixel is allotted for precise region. If none of the edges identified, cumulate adjacent pixel to a region class. Recapitulation is done for every frontier pixel in the ROI (Region of Interest). This approach is further concentrate on regions in Growing, Splitting and Merging [28].

In edge base loom, the edges are predictable and are associated to form region limits. It is the method of discovering evocative transitions in an image. Generally, the point where shrill changes in the brightness occur typically forms the border between different objects [29]. The various methods in edge based segmentation include Gradient operator [30], Prewitt Kernel [31], Sobel Kernel [32], Robert's Kernel, Laplacian of Gaussian and Canny edge detector [33].

Threshold, a modus operand in which the grayscale images are converted to binary based on a particular threshold value. There are three approaches in thresholding. They are: Adaptive Thresholding [34], Global Thresholding[35], and Histogram based Thresholding [36]. 


\subsubsection{Adaptive Threshold}

Irregular illumination makes an initially totally segmental image into an image that can't be portioned acceptably utilizing a solitary limit. One approach to defeat the irregular illumination issue is to initially assess the irregular light and correcting it appropriately. Upon remedy, Global thresholding can be utilized [37]. Another direction is to utilize versatile thresholding by dividing the first image into a few sub images and use Global thresholding strategies for each sub picture.

\subsubsection{Global Thresholding}

It is the least complex and most broadly utilized of all the possible partition techniques. In this technique, a limit is chosen with the end target that it is universally optimal. Division is done dependent on the edge esteem. An edge is supposed to be around the global optimal, if the quantity of misclassified pixels is least. Otsu's strategy comes under Global Thresholding.

\section{TABLE 2: OUTPUTS OF PROCESSING THE FETAL ULTRASOUND IMAGE USING VARIOUS} ALGORITHMS

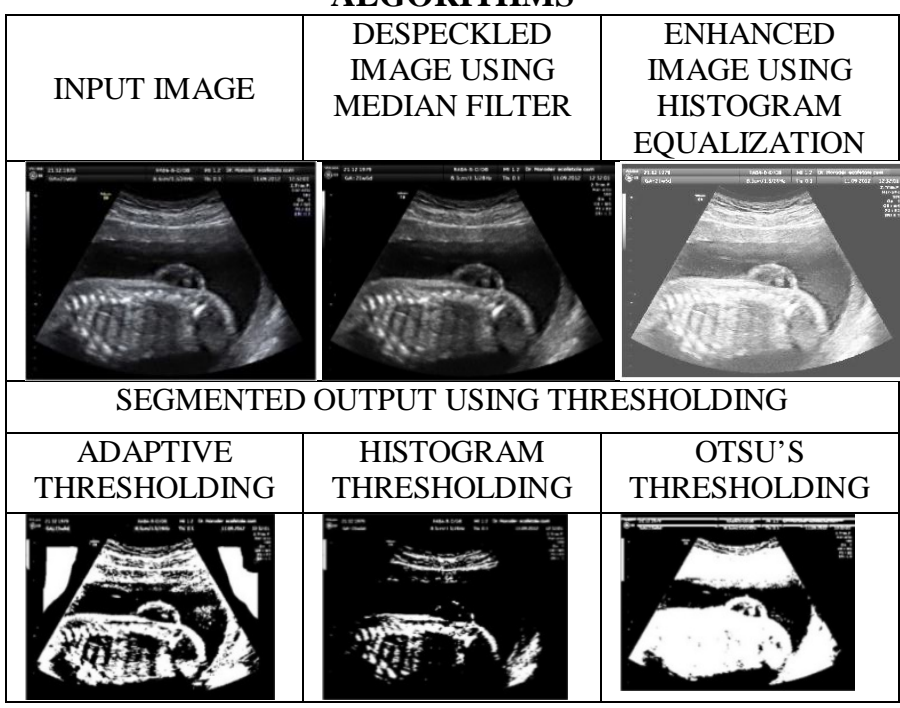

TABLE 3: PERFORMANCE MEASURES OF THE THRESHOLDING ALGORITHMS

\begin{tabular}{|c|c|c|c|}
\hline $\begin{array}{c}\text { SEGMENT } \\
\text { ATION } \\
\text { METHOD }\end{array}$ & $\begin{array}{c}\text { ADAPTIVE } \\
\text { THRESHOLD } \\
\text { ING }\end{array}$ & $\begin{array}{c}\text { HISTOGRAM } \\
\text { THRESHOLD } \\
\text { ING }\end{array}$ & $\begin{array}{c}\text { OTSU'S } \\
\text { THRESHOLD } \\
\text { ING }\end{array}$ \\
\hline TP & 41 & 10 & 1452 \\
\hline TN & 18308 & 29080 & 69170 \\
\hline FP & 985 & 524 & 200 \\
\hline FN & 751 & 1058 & 2448 \\
\hline P & 0.04 & 0.0187 & 0.8789 \\
\hline R & 0.0518 & 0.0094 & 0.3723 \\
\hline S & 0.9489 & 0.9823 & 0.9971 \\
\hline A & 0.9136 & 0.9484 & 0.9638 \\
\hline C & 0.4442 & 0.5304 & 0.7856 \\
\hline SSIM & 0.3777 & 0.4108 & 0.6409 \\
\hline
\end{tabular}

\subsubsection{Histogram - based Thresholding}

Assume that grey level histogram compares to an image, $f(x, y)$, made out of grey values in a light foundation, so that region and foundation pixels have dark levels assembled into two prevailing modes. One evident approach to extricate the region from the foundation is to choose a limit ' $\mathrm{W}$ ' that isolates these modes. At that point any point $(x, y)$ for which $f(x, y)>$ $\mathrm{W}$ is called an region point, in any case, the fact is known as a foundation point. On the off chance that two prevailing modes portray the image histogram, it is known as a bimodal histogram. Just a single limit is sufficient for separating the image. The yields of different division calculations are arranged in Table 2 .

The performances of various segmentation algorithms are measured in terms of Precision, Recall, Specificity, Accuracy, Correlation coefficient and Structural Similarity Index as in Table 3.

TP (True positive), TN (True negative), FP (False positive) and FN (False negative) are validation metrics used for assessing the eminence of a segmented image. In this scenario, our need is to match up to a segmented image with ground truth value. We can make supposition of taking forefront as "white" and environment as "black" pixels in ground truth.

True-Positive (“TP”) : Accurately segmenting as forefront False-Positive ("FP") : Fallaciously segmenting as forefront True-Negative ("TN"): Accurately detecting as environment(background)

False-Negative ("FN"): pixels fallaciously detected as environment(background)

Above defined metrics helps in computing accuracy, precision, specificity and sensitivity as defined below:

Sensitivity: Optimistic examination of availability of expected characteristics and computed as specified in equation (6).

$$
\frac{T P}{(T P+F N)}
$$

Specificity: Optimistic examination of unavailability of expected characteristics and computed as specified in equation (7).

$$
\frac{T N}{(T N+F P)}
$$

Precision: Precision means exactness. It is also known as predictive positive value and computed as specified in equation (8).

$$
\frac{T P}{(T P+F P)}
$$

Accuracy: Accuracy of the segmented result is deliberate as specified in equation (9). 


$$
\frac{(T P+T N)}{(T P+T N+F P+F N)}
$$

Other than these measures, Co efficiency of Correlation and Similarity index are handed to validate segmentation efficiency.

TABLE 4: GRAPHS SHOWING COMPARISON OF THREE THRESHOLDING ALGORITHMS BASED ON PERFORMANCE MEASURES

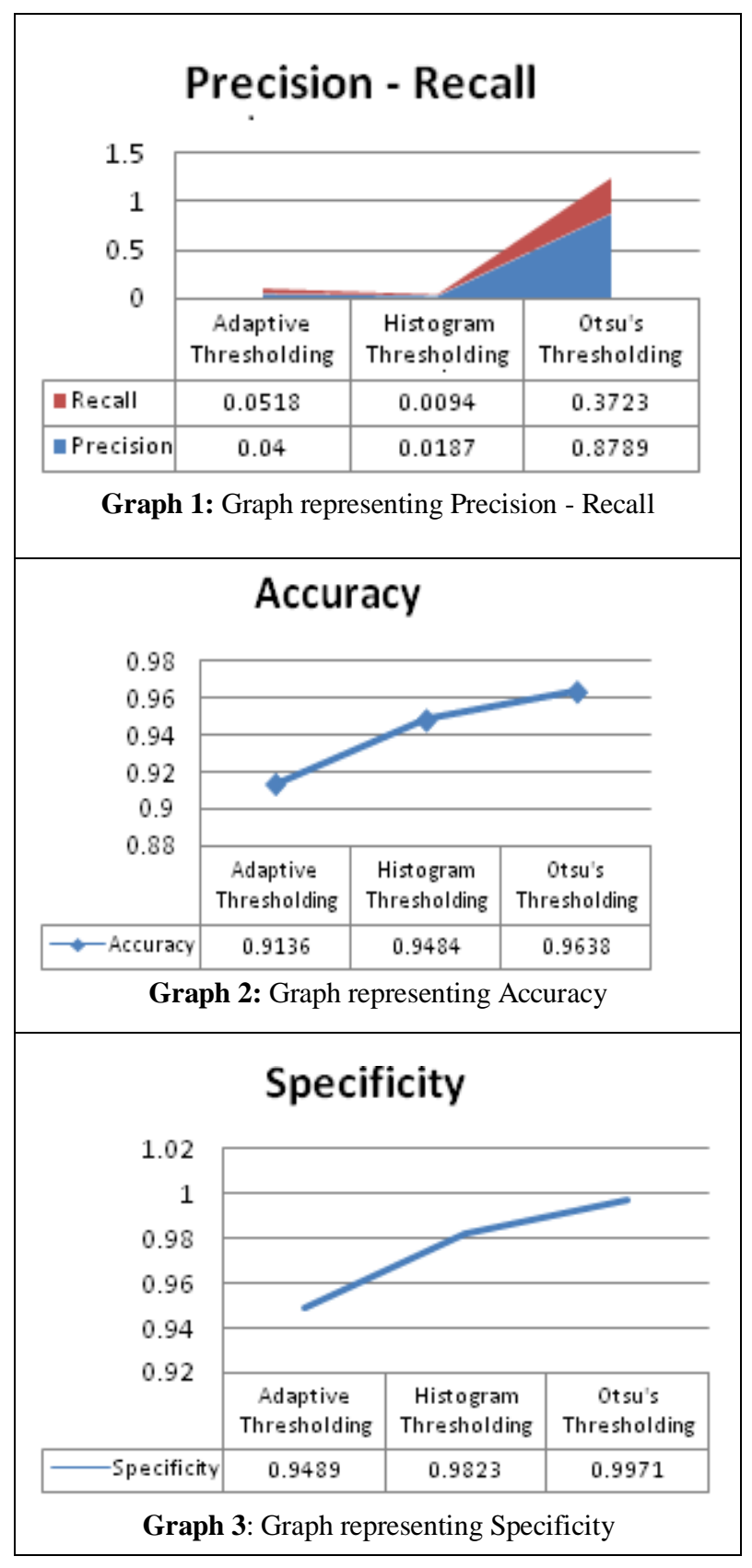

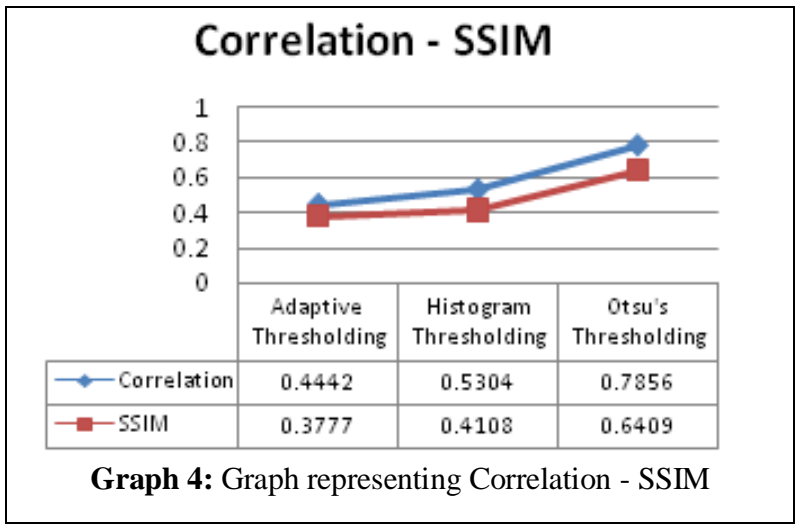

Correlation Coefficient: Correlation coefficient determines how much two signals or vectors are similar or different in phase and magnitude. Higher value of correlation co efficient indicates higher degree of similarity between the images. Correlation coefficient can be calculated as specified in equation (10).

$$
r=\frac{\sum_{m} \sum_{n}\left(\left(E_{m n}-\bar{E}\right)\left(F_{m n}-\bar{F}\right)\right)}{\sqrt{\left(\sum_{m} \sum_{n}\left(\left(E_{m n}-\bar{E}\right)^{2}\right)\left(\left(F_{m n}-\bar{F}\right)\right)^{2}\right)}}
$$

Where $\bar{E}$ represents mean of $\mathrm{E}$ and $\bar{F}$ represents mean of $\mathrm{F}$

SSI-Structural Similarity Index: The structural similarity (SSIM) index is a modus operandi for foreseeing the observed eminence of descriptions. SSIM assess likeness of two images. SSIM planned to advance up on customary methods called Peak Signal-to-Noise Ratio (PSNR) and Mean Squared Error (MSE).

The Structural Similarity (SSIM) Index distinction evaluation record as specified in equation (11) depends on the estimation of three terms, to be specific the luminance term, the differentiation term and the auxiliary term. The general list is a multiplicative blend of the three terms as specified in equation (12), (13) and (14).

$$
\begin{aligned}
& \operatorname{SSIM}(\mathrm{a}, \mathrm{b})=[\mathrm{I}(\mathrm{a}, \mathrm{b})]^{\alpha} \cdot[\mathrm{c}(\mathrm{a}, \mathrm{b})]^{\beta} \cdot[\mathrm{s}(\mathrm{a}, \mathrm{b})]^{\vee} \\
& \text { Wherel }(a, b)=\frac{2 \mu_{a} \mu_{b}+c_{1}}{\mu_{a}^{2}+\mu_{b}^{2}+c_{1}} \\
& c(a, b)=\frac{2 \sigma_{a} \sigma_{b}+\mathrm{c}_{2}}{\sigma_{\mathrm{a}}^{2}+\sigma_{\mathrm{b}}^{2}+\mathrm{c}_{2}} \\
& \mathrm{~s}(\mathrm{a}, \mathrm{b})=\frac{\sigma_{\mathrm{ab}}+\mathrm{c}_{3}}{\sigma_{\mathrm{a}}+\sigma_{\mathrm{b}}+\mathrm{c}_{3}}
\end{aligned}
$$

Based on the efficiency measures from Table 4, for our work we choose global thresholding method that separates the two peaks of image based on the intensity. Otsu's method finds minimized class variance and maximizes their combined spread.

This method automatically operates on the histogram directly and is fast. It assumes the histogram to be bimodal foreground and background pixels. Class variance with weighted value is computed as specified in equation (15).

$$
\sigma_{w}^{2}(t)=q_{1}(t) \sigma_{1}^{2}(t)+q_{2}(t) \sigma_{2}^{2}(t)
$$


Where, Class probabilities are estimated as specified in equation (16).

$q_{1}(t)=\sum_{i=1}^{t} P(i) q_{2}(t)=\sum_{i=t+1}^{I} P(i)$

Class means are known as specified in equation (17),

$\mu_{1}(t)=\sum_{i=1}^{t} \frac{i P(i)}{q_{1}(t)} \quad \mu_{2}(t)=\sum_{i=t+1}^{I} \frac{i P(i)}{q_{2}(t)}$

And Individual class variances are estimated in equation (18) and (19),

$$
\begin{aligned}
& \sigma_{1}^{2}(t)=\sum_{i=1}^{t}\left[i-\mu_{1}(t)\right]^{2} \frac{P(i)}{q_{1}(t)} \\
& \sigma_{2}^{2}(t)=\sum_{i=t+1}^{l}\left[i-\mu_{2}(t)\right]^{2} \frac{P(i)}{q_{2}(t)}
\end{aligned}
$$

Presently we go through the full scope of t esteems [1,256] and pick the worth that limits as specified in equation (20)

$$
\sigma_{w}^{2}(t)
$$

Algorithm 3 describes steps implicated in soliciting Global thresholding-Otsu's Method to an enhanced image.

Input : 2D matrix of histogram equalized image $m * n$.

Method : Minimal interclass variance is found using which the image is segmented

Output : 2D matrix of Segmented Binary image $m * n$.

Algorithm 3: Otsu's Thresholding Method

1. Every level intensity probabilities and histogram of image is evaluated.

2. Set the initial value of probability of class $\omega_{i}(0)$ and mean of class $\mu_{\mathrm{i}}(0)$.

3. Increment the threshold value from 1 to maximum intensity value and update $\omega_{\mathrm{i}}$ and $\mu_{\mathrm{i}}$.

4. For every iteration, the interclass variance $\sigma_{b}^{2}(t)$ is calculated.

5. The desired threshold value for Otsu's method corresponds to the minimum value of inter-class variance.

6. Binary image is formed by considering threshold value.

\subsection{FEATURE EXTRACTION}

It is yet another important phase in processing an image. A feature is the main characteristic that captures certain properties of the image. The segmented images are given as input to this phase. An best possible feature set should have effectual and discerning features; while generally reduce the redundant feature space [38]. There are various features that could be drawn out from image. In our work, we're going to concentrate on the extraction of morphological features [39] from the fetal ultrasound images in order to discern the fetal aberration.

A perfect list of capabilities should have successful and segregating highlights; while generally diminish the excess of highlight space [38]. There are different highlights that could be removed from a picture, for example, textural highlights, morphological highlights, model based highlights, descriptor highlights.

\subsection{FEATURE CLASSIFICATION}

SVM (Suport Vector Machine), supervised learning procedure which has to be trained with sample dataset before classification. During classification, the input images are compared or mapped with the features of the training dataset and are then classified. The objective of SVM is to locate the ideal isolating hyperplane which enhance the edge of training information. Given a specific hyperplane, we can evaluate the partition between the hyperplane and the nearest datapoint. In the event that we twofold this separation, we get an edge. The hyperplane with widest margin is the best possible separating hyperplane. The equation of an hyperplane is defined in equation (21),

$$
\mathrm{w}^{\mathrm{T}} \mathrm{x}=0
$$

\subsubsection{Binary Classification}

In order to classify the feature extracted spine and abdominal images as normal or abnormal, we go for SVM.

The SVM calculation looks for an best hyperplane that isolates the information into two classes. For separable classes, the perfect hyperplane augments an edge (space that doesn't contain any perceptions) encircle itself, which makes limits for the positive and negative classes. For indivisible classes, the goal is the equivalent, yet the calculation forces a punishment on the length of the edge for each perception that is on an inappropriate side of its group limit.

The Linear SVM score is computed as specified in equation (22),

$$
f(x)=x^{\prime} \beta+b
$$

Where, $\mathrm{x}$ is a perception (comparing to a column of $\mathrm{X}$ ).

The vector $\beta$ contains the coefficients that characterize a symmetrical vector to the hyperplane (comparing to Mdl.Beta). For divisible information, the ideal edge length is $2 /\|\beta\|$.

Where $\mathrm{b}$ is the predisposition term (comparing to Mdl.Bias).

The base of $f(x)$ for specific coefficients characterizes a hyperplane. For a specific hyperplane, $\mathrm{f}(\mathrm{z})$ is distance from point $\mathrm{z}$ toward the hyperplane.

The calculation looks for the greatest edge length, while keeping perceptions in the positive $(\mathrm{y}=1)$ and negative $(\mathrm{y}=-$ 1) classes independent. 


\subsubsection{Multiclass Classification}

After extracting the features of an abnormal image, we've to discern the type of structural aberration say Spina Bifida Occulta or Meningocele or Myelomeningocele in case of Spinal Ultrasound input and Exomphalos major or Exomphalos minor in case of Abdominal ultrasound input. For this classification, we go for SVM Multiclass Classification.

ECOC (error-correcting output code) multiclass model is utilized in Multiclass SVM which lessens the issue of characterization with at least three classes to a lot of twofold classifiers. ECOC order by and large requires a coding plan, which decides the classes that the parallel students train on, and an interpreting plan, which decides how the outcomes (forecasts) of the double classifiers are amassed. A coding configuration is where components direct which classes are prepared by every double student, that is, the way the multiclass issue is decreased to a progression of paired issues. Each line of the coding configuration relates to a particular class, and every segment compares to a twofold student. The different coding structures are: one versus one, one versus all, binary complete, ternary complete, ordinal, dense and sparse random.

\section{CONCLUSION}

In this paper, we've discussed about developing an automated decision support system to discern two structural aberrations in the fetus such as Spina Bifida, Exomphalos and then classify them based on their severity as Spina Bifida Occulta or Meningocele or Myelomeningocele and Exomphalos Major or Exomphalos Minor respectively. This is done by taking fetal ultrasound images of spine and abdomen as input and processing those using efficient algorithms. Future enhancement of this project could be routine based lesion recognition by enhanced 3D convolution neural networks (Dou et. al, 2020) and machine learning.

\section{REFERENCES}

1. UmutKonur.,Firket S Gurgen., FursonVarol., and LaleAkarun., (2015). Computer aided detection of Spina Bifida using nearest neighbour classification with curvature scale space features of fetal skulls extracted from ultrasound images. Elsevier knowledge based systems, Vol: 85, 80-95, doi: 10.1016/j.knosys.2015.04.021.

2. Athira P.K., Linda Sara Mathew, (2015). Fetal Anomaly Detection in Ultrasound Image. International Journal of Computer Applications, 129(9), 01-04, www.ijcaonline.org.

3. UmutKonur and Fikret S. Gürgen (2010). Computer Aided Diagnosis for Spina Bifida. IEEE Conference on Health Informatics and Bioinformatics, 104-109, doi: 10.1109/HIBIT.2010.5478897.

4. Mahmoud Al-Ayyoub and Duha Al-Zghool (2013). Determining the type of Long Bone Freacturess in X-Ray images, WSEAS Transactions on Information Science and Applications, 8(10), 261-270.
5. Cheng, H. D., Shan, J., Ju, W., Guo, Y., \& Zhang, L. (2010). Automated breast cancer detection and classification using ultrasound images: A survey. Pattern recognition, 43(1), 299-317.

6. Oleg V. Michailovich and Allen Tannenbaum (2006).Despeckling of Medical Ultrasound Images. IEEE Transactions on Ultrasonics, Ferroelectrics, and Frequency control, 53(1), 64-78, doi: 10.1109/TUFFC.2006.1588392.

7. Mu-Hsein Hsieh, Fan-Chieh Cheng, Mon-ChauShie, Shanq-Jang Ruan (2013).Fast and Efficient Median Filter for removing 1-99\% of Salt and Pepper noise in images. Elsevier - Engineering Applications of Artificial Intelligence, 26(4), 1333-1338, doi: 10.1016/j.engappai.2012.10.012.

8. Bedi S.S., RatiKhandelwal, (2013).Various Image Enhancement Techniques- A Critical Review. International Journal of Advanced Research in Computer and Communication Engineering, 2(3), 1605-1609, www.ijarcce.com.

9. Nikhil J. Dhinagar and Mehmet Celenk (2012). Ultrasound Medical Image Enhancement and Segmentation Using Adaptive Homomorphic Filtering and Histogram Thresholding, IEEE EMBS International Conference on Biomedical Engineering and Sciences, 349-353, doi: 10.1109/IECBES.2012.6498021.

10. Qureshi, Q. A., Khan, I., Shah, B., Nawaz, A., Waseem, M., \& Muhammed, F. (2014). Factors affecting the introduction of ICTs for Healthcare 'Decision-making' in hospitals of developing countries. Journal of Information Engineering and Applications. ISSN, 2224-5782.

11. Alison Noble J., DjamalBoukerroui, (2006). Ultrasound Image Segmentation: A Survey. IEEE Transactions on Medical Imaging, 25(8), 987-1010, doi: 10.1109/TMI.2006.877092.

12. Hetal J. Vala, AsthaBaxi (2013). A Review on Otsu Image Segmentation Algorithm, International Journal of Advanced Research in Computer Engineering \& Technology (IJARCET), 2(2), 387-389, www. ijarcet.org.

13. HimanshuMakkar, AdityaPundir (2014). Image Analysis Using Improved Otsu's Thresholding Method. International Journal on Recent and Innovation Trends in Computing and Communication, 2(8), 2122-2126, www.ijritcc.org.

14. Joel T., and Sivakumar R., (2013).Despeckling of Ultrasound Medical Images: A Survey. Journal of Image and Graphics, 1(3), 161-165, doi: 10.12720/joig.1.3.161-165.

15. Khairudin M., Irmawati D., (2015). Comparison Methods of Noise Elimination for

Pregnancy Image Processing, Int. Conference on Information Technology, Computer and Electrical Engineering (ICITACEE), 262-265, doi: 10.1109/ICITACEE.2015.7437810.

16. Vikrant Bhateja, KartikeyaRastogi, AviralVerma, ChiragMalhotra (2014).A Non-Iterative Adaptive Median Filter for Image Denoising. IEEE 
International Conference on Signal Processing and Integrated Networks (SPIN), 113-118, doi: 10.1109/ICIP.2005.1529701.

17. MandeepKaur, KiranJain,Virender Lather (2013). Study of Image Enhancement Techniques: A Review, International Journal of Advanced Research in Computer Science and Software Engineering, 3(4), 846-848, www.ijarcsse.com.

18. Kuldeep Singh, Rajiv Kapoor, Sanjeev Kr. Sinha (2015). Enhancement of low Exposure Images via Recursive Histogram Equalization Algorithms, Elsevier - International Journal for Light and Electron Optics, 126(20), 2619-2625 ,doi: 10.1016/j.ijleo.2015.06.060.

19. Sheng Hoong Lim, NorAshidi Mat Isa, Chen HeeOoi, Kenny KalVinToh (2013). A new histogram equalization method for digital image enhancement and brightness preservation, Article on Signal Image and Video Processing, Springer, 9(3), doi: 10.1007/s11760-013-0500-z.

20. Ravindra Pal Singh and Manish Dixit (2015). Histogram Equalization: A Strong Technique for Image Enhancement, International Journal of Signal Processing, Image Processing and Pattern Recognition 8(8), 345-352, doi: 10.14257/ijsip.2015.8.8.35.

21. AlirezaNorouzia, MohdShafry, MohdRahima, AymanAltameemb,

TanzilaSabac,

AbdolvahabEhsaniRada,

AmjadRehmand\&MueenUddine (2014). Medical Image Segmentation Methods, Algorithms, and Applications, IETE Technical Review Publication, 199-213, doi: 10.1080/02564602.2014.906861.

22. RohanKandwal, Ashok Kumar, Sanjay Bhargava (2014). Review: Existing Image Segmentation Techniques, International Journal of Advanced Research in Computer Science and Software Engineering 4(4), 153-156, www.ijarcsse.com

23. Mohammed. M. Abdelsamea (2011). An Automatic Seeded Region Growing for 2D Biomedical Image Segmentation, Proceedings of International Conference on Environment and Bio-Science, 01-05, doi: arXiv preprint arXiv:1412.3958.

24. NadeemMahmood, Asadullah Shah, Ahmad Waqas, AdamuAbubakar, Shafia Kamran, Syed BaqarZaidi (2015). Image Segmentation Methods And Edge Detection: An Application To Knee Joint Articular Cartilage Edge Detection, Journal of Theoretical and Applied Information Technology, 71(1), 87-96, www.jatit.org.

25. Yogamangalam R., Karthikeyan B., (2013). Segmentation Techniques Comparison in Image Processing, International Journal of Engineering and Technology, 5(1), 307-313.

26. Felzenszwalb, P.F., Huttenlocher, D.P., (2004).Efficient graph-based image segmentation, International Journal of Computer Vision, 59(2), 167-181, www.cs.brown.edu.

27. Nima Torbati, Ahmad Ayatollahi', Ali Kermani, (2014), An efficient neural network based method for medical image segmentation, Elsevier Computers in Biology and Medicine, 44(1), 76-87, doi:

http://dx.doi.org/10.1016/j.compbiomed.2013.10.02 9

28. Dinesh D. Patil1 ,Sonal G. Deore, (2013). Medical Image Segmentation: A Review, International Journal of Computer Science and Mobile Computing, 2(1), 22-27, www.ijcsmc.com.

29. Rashmi ,Mukesh Kumar and RohiniSaxena (2013). Algorithm And Technique On Various Edge Detection: A Survey, Signal \& Image Processing : An International Journal (SIPIJ), 4(3), 65-75, doi: 10.5121/sipij.2013.4306.

30. YinfeiZheng, Yali Zhou, Hao Zhou, and Xiaohong Gong (2015). Ultrasound Image Edge Detection Based on a Novel Multiplicative Gradient and Canny Operator, SAGE Journals - Ultrasonic Imaging, 37(3), 238-250, doi: $10.1177 / 0161734614554461$.

31. Sujatha P and Sudha K K., (2015).Performance Analysis of Different Edge Detection Techniques for Image Segmentation, Indian Journal of Science and Technology, 8(14), 01-06, doi: 10.17485/ijst/2015/v8i14/72946.

32. Subodh Kumar, PrabatPandey (2014). Implementation of X-Ray image segmentation by using edge detection based on Sobel Edge Operator, International Journal of Innovative Research \& Studies, 3(2), 190-202.

33. Manasa N., Mounica G., DivyaTejaswi B., (2016). Brain Tumor Detection Based on Canny Edge Detection Algorithm and it's area calculation, IEEE International Conference on Computer and Information Sciences (ICCOINS), doi: 10.1109/ICCOINS.2014.6868368

34. Jianlun Wang, Jianlei He, Yu Han, ChangquOuyang, Daoliang Li (2013). An Adaptive Thresholding algorithm of field leaf image, Elsevier - Computer and Electronics in Agriculture, 96, 23-39, doi: 10.1016/j.compag.2013.04.014

35. Kalavathi P (2013). Brain Tissue Segmentation in MR Brain Images using Multiple Otsu's Thresholding Technique, IEEE-International Conference on Computer Science \& Education (ICCSE 2013), 639-642, doi: 10.1109/ICCSE.2013.6553987.

36. Daniel RatnaRaju P and Neelima G (2012). Image Segmentation by using Histogram Thresholding, International Journal of Computer Science \& Engineering Technology, 2(1), 776-779, www.ijcsnet.net

37. Dou, Q., Chen, H., Qin, J., \&Heng, P.-A. (2020). Automatic lesion detection with three-dimensional convolutional neural networks. Biomedical Information Technology, 265-293. doi:10.1016/b978-0-12-816034-3.00009-2 
38. Cherkassky V S., Mulier F., (1998). Learning from Data: Concepts, Theory, and Methods, Wiley, New York, NY, USA

39. EyupEmreUlku, Ali YilmazCamurcu (2013). Computer Aided Brain Tumor Detection with Histogram Equalization and Morphological Image Processing Techniques, IEEE - International Conference on Electronics, Computer and Computation, 48-51, doi: 10.1109/ICECCO.2013.6718225. 